\title{
ANTROPOMETRÍA. ANÁLISIS COMPARATIVO DE LAS TECNOLOGÍAS PARA LA CAPTACIÓN DE LAS DIMENSIONES ANTROPOMÉTRICAS
}

\author{
$\checkmark$ ROSMERY NARIÑO LESCAY ${ }^{1}$ \\ Alicia Alonso BECERRA ${ }^{1}$ \\ ANAISA HERNÁNDEZ GONZÁLEZ ${ }^{1}$
}

\section{RESUMEN}

Este artículo constituye una revisión de los principales aspectos de la Antropometría y las distintas tecnologías para la captación de las dimensiones antropométricas. La antropometría es una disciplina científica que está estrechamente relacionada con la Ergonomía Física y se desarrolla en diferentes campos de aplicación. Es la ciencia que estudia las dimensiones del cuerpo humano, los conocimientos y técnicas para llevar a cabo las mediciones, así como su tratamiento estadístico.

Para obtener datos antropométricos con fines ergonómicos, ya sea para un estudio real o académico, es necesario contar con herramientas adecuadas para realizar este tipo de mediciones.

Son diversas las tecnologías o dispositivos existentes para la captación de las dimensiones antropométricas y aunque históricamente la antropometría ha sido unidimensional, registrada de forma manual, utilizando diferentes instrumentos tales como: estadiómetro, antropómetro, compás antropométrico, cinta métrica, silla antropométrica, entre otros; el desarrollo de las tecnologías ha permitido la creación de sistemas de captación de las dimensiones antropométricas que no requieren del contacto directo con la persona a medir.

PALABRAS CLAVES: Antropometría; tecnologías de medición; dimensiones antropométricas; análisis.

\section{ANTHROPOMETRY. COMPARATIVE ANALYSIS OF TECHNOLOGIES FOR THE CAPTURE OF ANTHROPOMETRIC DIMENSIONS}

\section{ABSTRACT}

This article is a review of the main aspects of anthropometry and various technologies for the capture of the anthropometric dimensions. Anthropometry is a scientific discipline that is closely related to Physical Ergonomics and

1 Instituto Superior Politécnico José Antonio Echeverría, La Habana - Cuba

Autor de correspondencia: Nariño Lescay, R. (Rosmery): Calle 17 \# 1405 e/ 26 y 28 apto. 12 Vedado, La Habana, Cuba. Teléfono: 5352728967. Correo electrónico: rosmery@dcrhmail.cujae.edu.cu
Historia del artículo:

Artículo recibido: 19-I-2016 / Aprobado: 23-XI-2016

Disponible online: 30 de febrero de 2017

Discusión abierta hasta abril de 2018 
develops in different fields of application. The science studies the dimensions of the human body, the knowledge and skills to perform measurements, as well as their statistical treatment.

In order to obtain anthropometric data for ergonomic ends, be it for actual or academic studies, it is necessary to have the appropriate tools for the realization of these types of measurements. There are various existing technologies and devices for capturing the anthropometric dimensions and anthropometry and although, historically, anthropometry has been one dimensional and manually registered using different instruments such as, stadiometer, anthropometer, anthropometric compass, measuring tape, anthropometric chair, among others, the development of technologies has enabled the creation of systems to capture anthropometric dimensions that do not require direct contact with the person being measured.

KEYWORDS: Anthropometry; Measurement technologies; Anthropometric dimensions; Analysis.

\section{ANTROPOMETRIA. ANÁLISE COMPARATIVA DAS TECNOLOGIAS PARA A CAPTAÇÃO DAS DIMENSÕES ANTROPOMÉTRICAS}

\section{RESUMO}

Este artigo é uma revisão dos principais aspectos da antropometria e as diferentes tecnologias para a captação das dimensões antropométricas. A antropometria é uma disciplina científica que está intimamente relacionado com a Ergonomia Física e se desenvolve em diferentes campos de aplicação. É a ciência que estuda as dimensões do corpo humano, os conhecimentos e as técnicas para realizar medições e seu tratamento estatístico. Para obter dados antropométricos para fins ergonômicos, tanto para o estudo real ou acadêmico, é necessário ter as ferramentas adequadas para realizar este tipo de medição.

Existem várias tecnologias ou dispositivos para capturar as dimensões antropométricas e embora historicamente a antropometria tem sido unidimensional, registrada manualmente, utilizando várias ferramentas, tais como: estadiômetro, antropômetro, bússola antropométrica, fita métrica, cadeira antropométrica, entre outros; o desenvolvimento das tecnologias tem permitido a criação de sistemas para capturar dimensões antropométricas que não requerem o contacto direto com a pessoa a ser medida.

PALAVRAS-CHAVE: Antropometria; Tecnologias de medição; Dimensões antropométricas; Análise.

\section{LA CAPTACIÓN DE LAS DIMENSIONES ANTROPOMÉTRICAS}

\section{INTRODUCCIÓN}

El conocimiento de las capacidades psicofisiológicas y antropométricas de los seres humanos es necesario para la realización de estudios ergonómicos que permitan evaluar y diseñar diferentes puestos de trabajo que se encuentren acordes a las características de las personas.
La antropometría, con fines ergonómicos, busca brindar datos antropométricos que sirvan como base para dimensionar objetos que se ajusten a las verdaderas características de los usuarios finales (Gómez, 2005). Es una disciplina científica que está estrechamente relacionada con la Ergonomía Física y se desarrolla en diferentes campos de aplicación.

Generalmente el trabajador debe adaptarse a "lo que ya existe", esto se debe principalmente a que gran parte de los mobiliarios son importados o no fueron diseñados para ser utilizados por los trabajadores. Hoy en día la mayoría de las tareas laborales 
requieren que el trabajador mantenga una postura fija por periodos de tiempos prolongados, si a esto se le adiciona un puesto mal diseñado, o sea, que no se corresponda con las características antropométricas de los usuarios finales, puede alentar la adopción de posturas incómodas, esfuerzos indebidos, provocando incomodidad, malestar y afectaciones en la salud de los trabajadores.

A raíz de lo antes expuesto fueron consultadas diferentes investigaciones en materia de evaluación ergonómica a puestos de trabajo. Con el resultado obtenido en estas investigaciones fue posible constatar que existen problemas de salud asociados al mal diseño de los puestos de trabajo lo que afecta la productividad, la eficiencia y la eficacia (Carmona, 2009; Díaz, 2009; Lubián, 2011; Gallardo, 2011; Barrabí, 2012; Martínez, 2012).

Se evaluó un total de 84 puestos, en diferentes empresas cubanas, utilizando herramientas de evaluación ergonómica como: Evaluación del Riesgo Individual (ERIN), Rapid EntireBodyAssessment (REBA), Rapid UpperLimbAssessment (RULA) y se encuestó un total de 319 trabajadores con el Cuestionario de Síntomas Músculo-Esqueléticos. Los resultados de estas evaluaciones arrojaron que 65\% de los puestos presentan alto riesgo, con necesidad de realizar cambios en breve período de tiempo, y que $35 \%$ requieren cambio en el diseño. Con el cuestionario aplicado se demostró la presencia de síntomas músculo-esqueléticos expresando por el 92\% de los trabajadores encuestados, además de sentir dolor en alguna región del cuerpo y la exposición a factores de riesgo de Desórdenes MúsculoEsqueléticos (DME) es elevada.

En este trabajo se presenta el sustento teórico de los principales aspectos de la Antropometría, de los estudios antropométricos y un análisis críticocomparativo de las distintas tecnologías para la captación de las dimensiones antropométricas, así como algunos estudios realizados con cada uno de los dispositivos desarrollados.

\section{ANTROPOMETRÍA Y ESTUDIOS ANTROPOMÉTRICOS}

El término Antropometría se refiere al estudio de la medición del cuerpo humano en términos de las dimensiones del hueso, músculo, y adiposo (grasa) del tejido. La palabra antropometría se deriva de la palabra griega antropo, que significa ser humano y la palabra griega metron, que significa medida. El campo de la antropometría abarca una variedad de medidas del cuerpo humano. El peso, la estatura (altura de pie), longitud reclinada, pliegues cutáneos, circunferencias (cabeza, la cintura, etc.), longitud de las extremidades, y anchos (hombro, muñeca, etc.) son ejemplos de medidas antropométricas (Pate, Oria y Pillsburry, 2012; Vicente, 2015; Norton y Tim, 2012).

A partir de la definición anterior, se puede señalar que la antropometría estudia todas las medidas corporales (Geraldo, 2015).

La antropometría es una representación cuantitativa sistemática del individuo con el propósito de entender su variación física. La antropometría se utiliza para el diseño de ropa y equipos, por ejemplo, a través de técnicas antropométricas para establecer las dimensiones humanas (Nada, Zuhair y Nawal, 2014).

Según Arellano (2009) la antropometría es la ciencia de la determinación y aplicación de las medidas de cuerpo humano, tanto en reposo como en movimiento; estas medidas están determinadas por la longitud de los huesos, músculos y de la forma de las articulaciones.

La antropometría, con fines ergonómicos, busca brindar datos antropométricos que sirvan como base para dimensionar objetos que se ajusten a las verdaderas características de los usuarios finales (Gómez, 2005; Narváez, 2013).

Todos los autores consultados coinciden que la antropometría es la disciplina que estudia las dimensiones dinámicas y estáticas del cuerpo humano, los procedimientos y las técnicas para llevar a cabo las mediciones, el análisis estadístico; brindando datos 
que sirvan para diseñar los objetos teniendo en cuenta las características de los usuarios finales, dando cumplimiento al principio ergonómico de adaptar los medios de producción a los trabajadores.

Cuando el puesto de trabajo no se adecua a la antropometría del trabajador se provocan esfuerzos innecesarios, limitación de la circulación sanguínea, fatiga en determinados grupos musculares y diferentes dolencias, además disminuye la productividad, aumenta la probabilidad de errores, disminuye la calidad y aumenta el número de accidentes de trabajo, (Alonso, 2006; Vázquez, 2013; Narváez, 2013).

Las dimensiones del cuerpo son de dos tipos: estructurales y funcionales. Las estructurales son de la cabeza, troncos y extremidades en posición de pie o sentado. Mientras que las funcionales o dinámicas son aquellas en donde está involucrado el movimiento realizado por el cuerpo en una actividad específica (Yáñez, 2009b).

Es decir, que en la antropometría estática se toman las medidas del cuerpo humano colocando a la persona en una posición fija midiendo entre puntos anatómicos específicos, y en la dinámica las medidas del cuerpo humano en movimiento.

Las dimensiones relevantes del cuerpo humano más utilizadas con fines de diseño han sido recomendadas por autores como: Alonso (2006); Panero (2009); ISO (2010), entre otros.

Las dimensiones antropométricas varían en individuos pertenecientes a diferentes etnias, periodos de tiempo, edad, sexo, etc. (Alonso, 2006). Para el diseño de puestos de trabajo es necesario tener en cuenta a la población que lo utilizará.

En un artículo de Amanda, Nogués y Pinilla (2008) se hace referencia a los estudios antropométricos en Cuba. Desde una perspectiva histórica se alega que el primer estudio antropométrico realizado en Cuba fue practicado por el médico francés Henri Dumont (1824-1878), quien en los años del 1865-1866, efectuó 27 mediciones en 7 personas, de las cuales 4 eran del sexo masculino y 3 del femenino, todos del mismo origen poblacional y de raza negra.
Son diversos los estudios antropométricos realizados en Cuba, la mayoría de ellos orientados al estudio de la nutrición, el deporte, al crecimiento y desarrollo infantil y al diseño de mobiliario escolar en escuelas primarias. Siendo más escasos los estudios orientados al diseño de puestos de trabajo.

En la bibliografía consultada se constató la escasez de datos antropométricos en Latinoamérica (Gómez, 2005; Franco, 2005; Oliveira, 2011). Existen algunos estudios de esta ciencia en países como México, Colombia, Chile, Venezuela ofrecidos por Ávila y Prado, 1999) en su libro "Dimensiones Antropométricas de la población Latinoamericana", donde también incluyeron a Cuba. En las dos ediciones del libro, referencian el mismo estudio realizado por Dr. Antonio Martínez Fuentes de la Universidad de La Habana, donde se midieron 34 dimensiones antropométricas a una muestra de 583 trabajadoras cubanas de los sectores agropecuarios, industriales y comercio. No sería correcto utilizar este estudio con fines de diseño, debido a que la muestra seleccionada representa el 0,1\% de la población existente, según la Oficina Nacional de Estadística e Información (ONEI), en los sectores que fueron objeto de estudio (ONEI, 2012). Este tiene más de 35 años de efectuado y no cuenta con todas las dimensiones requeridas para la evaluación y diseño de puestos de trabajo según la ISO (2010), por ejemplo: altura de los ojos de pie, altura de los ojos sentado, alcance mínimo del brazo, entre otros. Es válido destacar que los estudios publicados por Ávila y Prado han sido los más abarcadores que se han realizado en Latinoamérica.

En Europa y América del Norte también se han realizado estudios en países como: Alemania, España, Italia, Inglaterra, Estados Unidos, entre otros.

La mayoría de los estudios antropométricos consultados, como los publicados por Vicente et al. (2011); Rojas (2013); Alacid, Muyor y López (2011); K. Chhagan (2012); González et al. (2012); Betancourt y Manuel (2011); Guerra y Oriendo (2013); Marinho, Del Vecchio, y Franchini (2011); Lera et al. (2014) y Gerando (2015), desarrollados tanto en Cuba como en el resto del mundo, se 
refieren a la ciencia del deporte y la nutrición, otra parte de ellos orientados al mundo de la moda, al diseño y evaluación del mobiliario escolar en escuelas primaria, entre otros.

\section{TECNOLOGÍAS PARA LA}

\section{CAPTACIÓN DE LAS DIMENSIONES ANTROPOMÉTRICAS}

Son diversas las tecnologías existentes para la captación de las dimensiones antropométricas y la forma en que se pueden obtener los datos antropométricos. Estos últimos se pueden adquirir en diversos formatos: unidimensionales (1D), bidimensionales (2D) y tridimensionales (3D). Los datos 1D consisten en estaturas, longitudes y perímetros de segmentos corporales. Permiten establecer el tamaño del cuerpo humano, pero no la forma. Los datos 2D consisten en siluetas o secciones corporales; son contornos formados por curvas o puntos $(\mathrm{x}, \mathrm{y})$. La antropometría 3D está formada por nubes de puntos con coordenadas $(\mathrm{x}, \mathrm{y}, \mathrm{z})$ que representan la superficie del cuerpo. Un ejemplo son los escaneados $3 \mathrm{D}$ del cuerpo, formados por nubes de puntos que suelen contener entre 20.000 y 300.000 puntos. La adquisición, el tratamiento y análisis de los datos aumenta considerablemente en complejidad desde los datos 1D a los datos 3D (Vicente, 2015).

Los métodos de medición antropométricos pueden ser directos e indirectos (Alonso, 2006). El método directo se basa en la obtención de las dimensiones antropométricas directamente de la persona a partir de los puntos antropométricos, haciendo uso de equipos e instrumentos - como los mencionados anteriormente-, los cuales escasean en nuestro país. El costo estimado de adquirir un módulo de estos equipos es de \$2 668 (MédicosNutricionales, 2013), lo que constituye una desventaja para Cuba en lo que se refiere a su adquisición.

Principales ventajas de utilizar instrumentos manuales:

- Permite medir todas las dimensiones antropométricas.
- La lectura de las medidas es de forma directa.

Principales desventajas de utilizar instrumentos manuales:

- Requiere de personal entrenado para realizar las mediciones.

- Las medidas se toman de una en una por cada uno de los sujetos.

- El tiempo de medición, registro y procesamiento de la información es extenso.

Con el paso de los años el desarrollo de las tecnologías ha permitido la creación de sistemas de captación de las dimensiones antropométricas sin necesidad de interactuar con la persona directamente (Método indirecto).

La antropometría digital 3D surgió con la idea de reducir el tipo de adquisición por sujeto, y que el escaneado se reduce a pocos segundos, y el software de procesado puede proporcionar las dimensiones antropométricas de forma automática, pudiendo obtenerse datos que sean necesarios en cualquier momento (Vicente, 2015).

Los escáneres 3D pueden ser de cuerpo completo o de alguna parte en concreto del cuerpo, como los pies o la cabeza. Los hay de diversos tipos, como los de tecnología de luz estructurada, fabricados por la empresa francesa Telmat Industrie (SYMCAD) o el desarrollado por la empresa Textile Clothingand Technology Corporation $\left(\mathrm{TC}^{2}\right)$. Como alternativa surgen los escáneres de proyección láser, que aunque son más caros, la precisión de la forma 3D resultante es mucho mayor y se utilizan en la mayoría de estudios antropométricos de España. Como ejemplo de estos escáneres tenemos los de Cyberware (USA) y Human Solutions (Alemania) (Vicente, 2015).

Algunos de los dispositivos utilizados en la tecnología de medición indirecta, sus características fundamentales, su principio de operación y sus aplicaciones se mencionan a continuación: 


\section{a) Equipo de termografía infrarroja (Arellano, 2009)}

Básicamente es una cámara detectora de imágenes termográficas dentro de las bandas de longitud de onda de 8 a $12 \mu \mathrm{m}$. La cámara opera según el principio de escaneo del objeto a medir, el cual se muestrea mediante un escáner reflectante bidimensional.

El escáner horizontal realiza la detección en líneas de 300 píxeles cada una, con una frecuencia de muestreo de $135 \mathrm{~Hz}$ (a la derecha y a la izquierda) y opera como oscilador resonante movido por un motor de corriente continua. El escáner vertical configura la imagen completa a partir de las diversas líneas.

Se capturan 200 líneas, siendo la secuencia de repetición de imágenes 1,25 Hz. También puede trabajar con 100 o con 50 líneas. 2015)

b) SYMCAD (Arellano, 2009; Vicente,

El SYMCAD no utiliza láser ni otro tipo de radiaciones nocivas. El dispositivo de captura de datos es fijo (no hay piezas en movimiento) dando resultados seguros y de fácil mantenimiento. Su tecnología patentada de adquisición en 3D se basa en la técnica de proyección de franjas con luz natural. Extrae las medidas peculiares delimitadas por marcadores dispuestos sobre puntos anatómicos, las cuales detecta e identifica automáticamente y calcula las medidas antropométricas adecuadas según las normas ISO-7250 e ISO 8559 (perímetros, alturas, longitudes...) o específicas.

\section{c) Body Scanner}

El 3D Body Scanner está compuesto por cuatro columnas, cada una de las cuales incluye dos cámaras CCD y un láser de categoría 1 inocuo para la vista. Las unidades se fijan en el suelo. Solo son necesarios 8 segundos para explorar la totalidad del cuerpo humano. Para ello, el cliente debe desvestirse y permanecer de pie en una postura natural (Investor's Business, 2014).
El sistema detecta la superficie del cuerpo humano y reproduce en la computadora una representación tridimensional.

Hasta aquí se han descrito los principales instrumentos que no requieren contacto físico con el usuario sugerido por Arellano (2009). Otros autores como Annichini et al. (2013); Overton (2013); Polviven (2012); Zwane, Moses y Lawrence (2010); Giachetti et al. (2015); Bing-ru et al. (2010), realizaron investigaciones con el uso de la tecnología 3D Body Scanner. Sin embargo en nuestro país, por cuestiones económicas, no es posible la adquisición de esta tecnología tan costosa. (Rodríguez, 2011).

Desde la aparición de los escáneres 3D se han realizado multitud de estudios antropométricos (Vicente, 2015). Se pueden citar estudios antropométricos con escáner 3D en diversos países como USA (Size USA), Francia (Estudio antropométrico de la población francesa), Alemania (SizeGermany), España (Estudio antropométrico de la población femenina en España), entre otros (Magros, 2012; Rodríguez, 2010.

Un estudio antropométrico realizado en México que comenzó a finales del 2010, con una muestra inicial calculada de 16,000 personas en las 14 ciudades más importantes del país, utilizó tecnología 3D Body Scanner y estimó inicialmente un costo de 500000 USD (Quezada, 2010). Al término del estudio se midieron 17364 personas de ambos géneros (Ruvalcaba, 2012). Las medidas fueron realizadas a personas de 18 a 66 años o más. La muestra tomada representa el 0,023\% de la población mexicana en ese rango de edades, y su objetivo fue ser usada por el sector de la confección para manufacturar las prendas de vestir, según las características anatómicas de los consumidores (Cherem, 2012).

Principales ventajas de utilizar a), b) y c) explicadas anteriormente:

- Solo son necesarios muy pocos segundos para explorar la totalidad del cuerpo humano.

- Permite realizar un gran número de mediciones en corto tiempo.

Revista EIA Rev.EIA.Esc.Ing.Antioq / Universidad EIA 
- La manipulación del individuo es mínima.

- Precisión y fidelidad de los resultados.

Principales desventajas de utilizar a), b) y c) explicadas anteriormente:

- Las personas deben trasladarse hacia el lugar donde esté instalada la cabina.

- Necesidad de capacitar personal para la utilización del mismo.

- Equipos costosos.

\section{d) Medición con una fotografía}

Esta técnica tampoco requiere del contacto físico con la persona a medir, se basa principalmente en la geometría proyectiva, la cual establece un modelo matemático de una cámara fotográfica de la forma: $\mathrm{m}=$ PM. Donde $\mathrm{m}$ es la imagen del objeto, $\mathrm{M}$ es el objeto a medir y P, es la matriz de proyección. Para realizar las mediciones es necesario tener un patrón de referencia (Yáñez, 2009a).

La geometría proyectiva es una alternativa en el diseño y desarrollo de software para realizar mediciones antropométricas de una forma muy simple a través de una cámara fotográfica (Yáñez, 2009a).

La presencia física de la persona deja de ser necesaria para el proceso de toma de medidas, y puede realizarse en cualquier momento y lugar con solo enviar un fichero con las fotografías para ser procesadas.

Actualmente esta técnica también es aplicada con la geometría epipolar con el uso de dos cámaras fotográficas.

La geometría epipolar es determinada por las correspondencias en los puntos. La selección y correspondencia de los puntos característicos en las dos vistas son el procedimiento estándar para recuperar la profundidad. El nombre de geometría epipolar es debido a que los puntos en los cuales la recta que une los centros de proyección de las cámaras corta a los planos de proyección se llaman epipolos (Moreno, 2008).
Esta técnica se está utilizando para determinar las dimensiones del cuerpo humano, dimensiones y reconocimiento de objetos y otros. La autora sigue investigando en la búsqueda de estudios antropométricos realizados con la aplicación de esta técnica.

Principales ventajas de utilizar la fotografía:

- La presencia física de la persona deja de ser necesaria para el proceso de toma de medidas. Puede realizarse en cualquier momento y lugar con sólo enviar un fichero con las fotografías para ser procesadas.

- Precisión y fidelidad de resultados.

Principales desventajas de utilizar la fotografía:

- Es necesario contar con un marco de referencia para tomar la fotografía.

- Es primordial una adecuada iluminación que permanezca constante durante todo el tiempo.

e) Kinect (medición con imágenes y movimiento)

Kinect, cuyo nombre originalmente fue "Proyecto Natal", es un dispositivo creado por Alex Kipman y desarrollado por Microsoft para su videoconsola Xbox 360 (Magros, 2012; Samaniego, 2012).

Este dispositivo fue anunciado por primera vez en junio de 2009 en la ElectronicEntertainment Expo 2009 como la nueva generación de entretenimiento en el hogar. Con el uso de la cámara se puede controlar mediante movimientos del cuerpo, las acciones del jugador y los menús de juego (Magros, 2012).

El dispositivo Kinect dispone de dos cámaras, RGB y NIR, y una fuente de luz infrarroja que permite que la cámara NIR obtenga sus datos incluso en ausencia de luz (Magros, 2012).

Mientras que la cámara RGB se encarga de obtener la información de color de todo aquello situado en su campo de visión, la cámara infrarroja de corto alcance se encarga de obtener la información de profundidad. Las dos lentes exteriores corresponden con la información infrarroja mientras que 
la lente situada en medio corresponde con la cámara a color (Magros, 2012).

Existen diferentes drivers para el uso de Kinect mediante un ordenador. Entre los que se encuentran Kinect for Windows SDK, OpenNI, OpenKinect o Libfreenect (Magros, 2012; Velardo y Dugelay, 2011; Lee et al., 2015; Clarkson et al., 2014).

Kinect SDK es capaz de detectar la posición de hasta seis personas, pero solo es capaz de registrar el esqueleto de dos de ellas (las dos más cercanas) obteniendo la estructura que contiene los 20 puntos detectados. Para poder identificar posturas y posiciones parcialmente ocultas, Kinect cuenta 200 posturas comunes para poder rellenar los espacios ocultos (Magros, 2012).

Este dispositivo ha sido aplicado en:

- Videojuegos para XBOX 360

- Asistente para aparcamiento de vehículos

- Desarrollo de prótesis ortopédicas

- Visualización en quirófanos

- Tratamiento parálisis cerebral

- Control del peso

- Estudios geográficos

- Control de robot mediante comandos corporales

Es una herramienta interactiva que actualmente se está utilizando para adquirir datos antropométricos. Este sensor pertenece a la clase de dispositivos conocida como las cámaras de profundidad. Comparado a muchos sistemas de captura de movimiento tradicionales, el Kinect no requiere que con anterioridad sean marcados los puntos de referencia en las personas que serán medidas. Este rasgo reduce tiempo de colección de datos substancialmente, pero también reduce la exactitud. Adquirir esta herramienta tiene un costo de aproximadamente 150 dólares (Espitia, Sánchez y Uribe, 2014; Robinson y Parkinson, 2013; Samaniego, 2012).

A pesar de ser una herramienta que aún está en desarrollo para la captación de datos antropométricos, se han realizados algunos estudios como los publicados por Velardo y Dugelay (2011); Lee et al. (2015) y Clarkson et al. (2014) donde se compara esta tecnología con el método tradicional.

Principales ventajas de utilizar esta tecnología:

- No requiere de que se marquen los puntos de referencia en los usuarios.

- Sensor económico.

- Facilidad de preparación en diferentes ambientes.

- Se conecta a una computadora.

\section{Principales desventajas:}

- El usuario debe realizar una serie de tareas que son comandas por el realizador del estudio.

- La computadora a la que se conecte debe contar con un driver específico para el uso del Kinect.

- El software de Kinect no permite rastrear el esqueleto usando más de un sensor al mismo tiempo.

\section{f) Aplicación Android para medi-} ción antropométrica desde móviles

Android es un sistema operativo inicialmente pensado para teléfonos móviles, pero que posteriormente se ha ido usando en tabletas y otros tipos de dispositivos como pueden ser electrodomésticos, televisores o relojes. Lo que lo hace diferente es que está basado en Linux, un núcleo de sistema operativo libre, gratuito y multiplataforma (Vicente, 2015).

Esta aplicación Android para móviles consiste en tomar dos fotografías, una de frente y otra de perfil de una persona que tendrá que colocarse como indican las siluetas que se mostrarán en la pantalla del teléfono y con técnicas de tratamiento de imagen se extrae el contorno de esa persona para cada fotografía. El smartphone fue el medio elegido tanto para la captura de imágenes como para su posterior tratamiento para obtener los datos necesarios para 
la reconstrucción 3D y las medidas antropométricas necesarias (Vicente, 2015).

Una vez reconstruido el modelo 3D, mediante librerías propias desarrolladas en el Instituto Biomecánica de Valencia (IBV), se calculan las medidas antropométricas necesarias que pueden servir tanto para la confección de ropa, para un futuro probador de ropa virtual o para futuras aplicaciones que se puedan desarrollar (Vicente, 2015).

Se realizaron comparaciones con 18 dimensiones obtenidas con la nueva aplicación y un Scanner 3D, existiendo diferencias más pequeñas y otras más grandes, como por ejemplo que en la altura a mitad del cuello el error porcentual no alcanza el $1 \%$ (1,46 mm de diferencia) y sin embargo en la lon- gitud de la entrepierna delantera sobrepasa el 35\% (86,8 mm de diferencia). El autor de esta aplicación afirma que el algoritmo para los cálculos de las dimensiones antropométricas puede mejorar, ya que a la hora de hacer el proyecto la versión disponible era la primera (Vicente, 2015).

Por ser un proyecto reciente aún no se cuenta con las posibles ventajas y desventajas que pueda tener esta aplicación.

En la Tabla 1 se muestra un resumen del análisis crítico-comparativo de las principales características de las tecnologías que han sido utilizadas para la medición o captación de las dimensiones antropométricas.

\begin{tabular}{|c|c|c|c|c|c|}
\hline DISPOSITIVOS & $\begin{array}{l}\text { MÉTODO DE } \\
\text { MEDICIÓN }\end{array}$ & PRECISIÓN* & DIMENSIONES & TIEMPO & COSTO (\$) \\
\hline $\begin{array}{l}\text { Instrumentos } \\
\text { Manuales }\end{array}$ & Directo & Poco preciso & $\begin{array}{c}\text { Todas las } \\
\text { dimensiones } \\
\text { antropométricas }\end{array}$ & $\begin{array}{l}45 \text { a } 60 \text { minutos } \\
\text { por sujeto }\end{array}$ & $\begin{array}{l}\text { Conjunto de } \\
\text { equipos } \\
2.668 \text { usd }\end{array}$ \\
\hline $\begin{array}{c}\text { (Láseres) } \\
\text { Termografía } \\
\text { infrarroja } \\
\text { SYMCAD } \\
\text { BodyScanner }\end{array}$ & Indirecto & Preciso & $\begin{array}{c}\text { Es posible captar } \\
\text { más de } 200 \\
\text { dimensiones }\end{array}$ & $\begin{array}{l}8 \text { a } 10 \text { segundos } \\
\text { por sujeto }\end{array}$ & Máx.: 35.000 usd \\
\hline $\begin{array}{l}\text { Medición con una } \\
\text { fotografía }\end{array}$ & Indirecto & Preciso & $\begin{array}{l}\text { No es posible } \\
\text { captar todas las } \\
\text { dimensiones } \\
\text { antropométricas } \\
\text { (circunferencias y } \\
\text { perímetros) }\end{array}$ & $\begin{array}{l}1 \text { a } 2 \text { minutos } \\
\text { por sujeto }\end{array}$ & $\begin{array}{l}89-1363 \text { usd } \\
\text { (depende del } \\
\text { modelo y la } \\
\text { marca) }\end{array}$ \\
\hline Kinect & Indirecto & Poco preciso & $\begin{array}{c}\text { Todas las } \\
\text { dimensiones } \\
\text { antropométricas } \\
\text { (depende de la } \\
\text { programación } \\
\text { del software) }\end{array}$ & $\begin{array}{l}1 \text { a } 2 \text { minutos } \\
\text { por sujeto }\end{array}$ & $150-250$ usd \\
\hline $\begin{array}{c}\text { Aplicación } \\
\text { Android para } \\
\text { medición } \\
\text { antropométrica } \\
\text { desde móviles } \\
\text { (Versión primera) }\end{array}$ & Indirecto & $\begin{array}{l}\text { Poco precisa } \\
\text { (aplicación } \\
\text { reciente, se } \\
\text { están realizando } \\
\text { estudios para } \\
\text { mejorar) }\end{array}$ & $\begin{array}{l}\text { Es posible captar } \\
\text { más de } 200 \\
\text { dimensiones }\end{array}$ & $\begin{array}{l}1 \text { a } 2 \text { minutos } \\
\text { por sujeto }\end{array}$ & $\begin{array}{l}\text { Está basado en } \\
\text { Linux, un núcleo } \\
\text { de sistema } \\
\text { operativo libre, } \\
\text { gratuito y } \\
\text { multiplataforma }\end{array}$ \\
\hline \multicolumn{6}{|c|}{ Fuente: Elaboración Propia } \\
\hline
\end{tabular}


Del análisis que se muestra en la Tabla 1 se considera que de las técnicas utilizadas, la medición manual a partir de instrumentos es la única que presenta contacto directo con el sujeto a medir. Del resto se puede destacar que el tiempo de medición es inferior. La técnica de medición con fotografía, a pesar de que no es posible captar las circunferencias y los perímetros, la autora considera que es posible captar las dimensiones necesarias para el diseño antropométrico de los puestos de trabajo; y del análisis realizado hasta el momento es la que brinda mayores posibilidades para esta investigación en Cuba dado su costo, la precisión que es posible lograr - constatada en los estudios con esta tecnología- y que no es necesario transportar a los sujetos a ser estudiados.

\section{CONCLUSIONES}

El análisis realizado a los diferentes aspectos de la Antropometría y a los estudios antropométricos en Cuba y el resto del mundo ayudaron a evidenciar la necesidad de contar con las dimensiones antropométricas de la población cubana, principalmente de la población laboral actual, la cual presenta afectaciones a la salud relacionadas fundamentalmente con el mal diseño de los puestos de trabajo.

Los estudios antropométricos cubanos desarrollados están dirigidos fundamentalmente a la ciencia del deporte, a la nutrición y al crecimiento y desarrollo infantil, aportándole un valor metodológico a la investigación doctoral que se basa en el diseño de un modelo para el estudio antropométrico y la evaluación y diseño de puestos de trabajo, así como una herramienta (software) para que aumente la eficiencia y la eficacia en la aplicación práctica del modelo. Además contribuye al perfeccionamiento de la impartición del laboratorio de antropometría de la asignatura de Ergonomía en los cursos de pregrado, postgrado y maestrías.

Para la realización de estudios antropométricos se utilizan: instrumentos manuales, la termografía infrarroja, el SYMCAD, el BodyScanner, la medición con fotografías, el Kinect y la aplicación Android para medición antropométrica desde móviles. En el análisis crítico comparativo de estas técnicas, tomando en consideración las dimensiones antropométricas que permiten captar, el costo de adquisición, el entrenamiento que requiere el personal que debe realizar las mediciones, la precisión, el tiempo de medición y la necesidad de transportar a los sujetos a ser estudiados, se considera que la tecnología para la captación de los datos antropométricos que en estos momentos ofrece las mayores posibilidades para esta investigación en Cuba es la medición a partir de la fotografía.

\section{REFERENCIAS}

Al-jassim, N.H.; Fathallah, Z.F.; Abdullah, N.M. (2014). Anthropometric Face in Basrah. Basrah Journal of Surgery, 20(2), pp. 29-40.

Alacid, F.; Muyor, J.M.; López Miñarro, P.A. (2011). Perfil antropométrico del canoísta joven de aguas tranquilas. International Journal of Morphology, 29, 835-840.

Alonso, A. (2006). Ergonomía, La Habana, Cuba.

Amada, J. L. N.; Nogués, J. Á.O.; Pinilla, S.B. (2008). Genes, ambiente y enfermedades en poblaciones humanas, Prensas Universitarias de Zaragoza.

Annichini, M.; Arena, R.; Fanini, M.; Fattorel, M.; Pavei, D.; Tasson, D.; Garro, V.; Lovato, C.; Giachetti, A. (2013). Shape processing for digital anthropometry.

Arellano, D.; Yáñez Mendiola, J. (2009). Mediciones Antropométricas sin contactos a partir de fotografías. Ide@s CONCYTEG, 48, pp. 669-673.

Avila, R.; Prado, L. (1999). Dimensiones antropométricas de la población Latinoamericana, México, Universidad de Guadalajara, Centro Universitario de Arte, Arquitectura y Diseño, División de Tecnología y Procesos, Departamento de Producción y Desarrollo, Centro de Investigaciones en Ergonomía.

Avila R.; Prado, L. (2007). Dimensiones antropométricas de la población Latinoamericana, México, Universidad de Guadalajara, Centro Universitario de Arte, Arquitectura y Diseño, División de Tecnología y Procesos, Departamento de Producción y Desarrollo, Centro de Investigaciones en Ergonomía.

Barrabí Núñez, R. (2012). Intervención ergonómica dirigida a la prevención de DMEs en la empresa de elaboración y empaque Chefpaq. Trabajo de Diploma, ISPJAE. 
Betancourt León, H., Aréchiga Viramontes, J.; Ramírez García, C. (2011). Proporcionalidad corporal de estudiantes cubanos de danza clásica, moderna y folclórica. Archivos de Medicina del Deporte, 28(142), pp. 93-102.

Bing-Ru, L.; Sun Shou, Q.; Rui Min, L.; Zhi Dong, Z.; Yang, L. (2010). Automatic measurement of scanned human body in fixed posture. IComputer-Aided Industrial Design \& Conceptual Design (CAIDCD), 2010 IEEE 11th International Conference on. IEEE 11th International Conference.

Carmona Ávila, E. (2009). Diseño de un procedimiento Ergonómico para la prevención de desordenes músculos-esqueléticos de origen laboral en empresas DATYS, sustentado por la Gestión de la Seguridad basado en conductas.Trabajo de Diploma, ISPJAE.

Chhagan, M.K.; Kauchali, S.; Van den Broeck, J. (2012). Clinical and contextual determinants of anthropometric failure at baseline and longitudinal improvements after starting antiretroviral treatment among South African children. Tropical Medicine and International Health, 17(9), pp. 1092-1099.

Clarkson, S.; Wheat, J.; Heller, B.; Choppin, S. (2014). Assessing the suitability of the Microsoft Kinect for calculating person specific body segment parameters. Computer Vision-ECCV 2014 Workshops, Springer, pp. 372-385.

Cherem, E.M. (2012). ¿Cuánto mide México? El tamaño no importa. CANAIVE Cámara Nacional de la Industria del Vestido.

Díaz Ferriols, Y. (2009). Diseño y Aplicación de un procedimiento para la identificación y evaluación de síntomas y factores de riesgo asociados con los desordenes músculo-esquelético de origen laboral en empresas cubanas. Trabajo de Diploma, ISPJAE.

Espitia-Contreras, A.; Sánchez-Caiman, P.; Uribe-Quevedo, A. (2014). Development of a Kinect-based Anthropometric Measurement Application. IEEE Virtual Reality. Minneapolis, Minnesota, USA, p. 2.

Franco Trujillo, J.; Quintana Ramírez, F.J.; Pañuelas Beltrán, E.; Anzaldo Juárez, P. (2005). Estudio antropométrico en trabajadores de Transportación Ferroviaria Mexicana. Rev Fac Med UNAM,48(3).

Gallardo Montes de Oca, D. (2011). Estudio ergonómico en la fábrica de transformadores latino. Trabajo de Diploma, ISPJAE.
Geraldo, A.P. (2015). Ergonomía y Antropometría aplicada con criterios ergonómicos en puestos de trabajo en un grupo de trabajadoras del subsector de autopartes en Bogotá, DC, Colombia. Revista Republicana, 2-3, pp. 135-150.

Giachetti, A.; Lovato, C.; Piscitelli, F., Milanese, C.; Zancanaro, C. (2015). Robust Automatic Measurement of 3D Scanned Models for the Human Body Fat Estimation. Biomedical and Health Informatics, IEEE Journal of, 19, pp. 660-667.

Gómez Parra, M.K. (2005). Sistemas de medición antropométrica para posturas sedentes (modelo funcional). Trabajo de grado presentado como requisito para optar al título de Diseñador Industrial, Universidad Industrial de Santander.

González Jiménez, E.; Aguilar Cordero, M.J.; Álvarez Ferre, J.; Padilla López, C.; Valenza, M.C. (2012). Estudio antropométrico y valoración del estado nutricional de una población de escolares de Granada; comparación con los estándares nacionales e internacionales de referencia. Nutrición Hospitalaria, 27(4).

Guerra, J.; Oriondo R. (2010). Estudio comparativo cineantropométrico de karatekas pertenecientes a la selección universitaria Inca Garcilaso de la Vega ya la Selección Peruana. Lima. Anales de la Facultad de Medicina, 2013. S18.

Investor's Business, D. (2014). Full-body scanners. Investors Business Daily, A02.

ISO (2010). Basic human body measurements for technological design - Part 1:Body measurement definitions and landmarks (ISO 7250-1:2010). Austrian Standards Institute.

Lee, H.-W.; Liu, C.-H.; Chu, K.-T.; Mai, Y.-C.; Hsieh, P.-C.; Hsu, K.-C.; Tseng, H.-C. (2015). Kinect Who's ComingApplying Kinect to Human Body Height Measurement to Improve Character Recognition Performance. Smart Science, 3(2), pp. 117-121.

Lara, L.; Albala, C.; Ángel, B.; Sánchez, H.; Picrin, Y.; Hormazabal, M.J.; Quiero, A. (2014). Predicción de la masa muscular apendicular esquelética basado en mediciones antropométricas en Adultos Mayores Chilenos. Nutrición Hospitalaria, 29, pp. 611-617.

Lubián Hernández, P. (2011). Estudio Ergonómico en los Laboratorios de Análisis Químico y Materiales del CEADEN. Trabajao de Dilploma, ISPJAE.

Magros Viforcos, E. (2012). Aplicación de las cámaras 3d al reconocimiento de actividades. Proyecto fin de Gra- 
do, Universidad Carlos III De Madrid. Escuela Politécnica Superior.

Marinho, B.F.; Del Vecchio, F.B.; Franchini, E. (2011). Condición física y perfil antropométrico de atletas de artes marciales mixtas. Revista de Artes Marciales Asiaticas, 6(2), pp. 7-18.

Martínez Vega, J. C. R. O., Alberto. (2012). Estudio de la organización del trabajo en el proceso productivo de la empresa Durero Caribe S.A. Trabajo de Diploma, ISPJAE.

Médicos-Nutricionales, B. E. (2013). Antropometría [Online]. México. [Online] Disponible en: http://bcequipos.com.mx/comercio/index.php 2014].

Moreno Escobar, J.J. (2008). Visión estéreo. Modelo ANOVA de bloques aleatorizados. Master de Informática Aplicada, Universidad Autónoma de Barcelona.

Narváez Morales, Y. A. (2013). Ergonomía y Antropometría. Más que Ciencias. Batutas para el diseño. Revista M.A, Mueble Actual. Sección: Diseño de Mobiliario. Artículo: La Ergonomía II Parte.

Norton, K.; Olds, T. (2012). Antropometría. Anthropometrica, Edición en Español: Dr. Juan Carlos Mazza ed. University of New South Wales Press, Sidney 2052 Australia.

Oliveira Damasceno, V.; Macedo Vianna, J.; Silva Novaes, J.; De Lima, J.P.; Miguel Fernandes, H.; Machado Reis, V. (2011). Relationship between anthropométrie variables and body image dissatisfaction among fitness center users. Revista de Psicología del Deporte, 20, pp. 367-382.

ONEI, Oficina Nacional de Estadística e Información, República de Cuba. (2012). Censo de Población y Viviendas 2012. Informe nacional. Resultados definitivos de indicadores seleccionados en Cuba, provincias y municipios. La Habana. [Online] Disponible en: http://www.one.cu/informenacional2012.htm [Consultado 6 de mayo de 2014].

Overton, G. (2013). Will full-body scanners keep you safe and secure? Laser Focus World, 49, pp. 45-47.

Panero, J. (2009). Las Dimensiones Humanas en los espacios Interiores, Editorial Félix Varela, La Habana.

Pate, R.; Oria, M.; Pillsbury, L. (2012). Fitness Measures and Health Outcomes in Youth. Committee on Fitness Measures and Health Outcomes in Youth; Food and Nutrition Board; Institute of Medicine. Washington (DC): National Academies Press (US).
Washington (DC): National Academies Press (US); 2012 Dec 10.

Polviven, E. (2012). [TC]2, Textile Clothing technology Corporation: 3D Body Scanning Technologies. Intellifit Bodyscan Technology for Public Sizing and Fit Services [Online]. [Consultado 3 de mayo de 2014].

Quezada, R. (2010). Cuánto mide México [Online]. Mexico: 2010. [Online] Disponible en: http://exp.mx/ n002M30 [Consultado 3 de mayo de 2014].

Robinson, M.; Parkinson, M. (2013). Estimating Anthropometry with Microsoft Kinect.

Rodríguez, I. (2010). Ropa exclusiva para mexicanos [Online]. Mexico: 2010. [Online] Disponible en: http:// exp.mx/n002PUB [Consultado 3 de mayo de 2014].

Rodríguez, I. (2011). Productos a la medida de los mexicanos [Online]. México: 2011. [Online] Disponible en: http://www.cnnexpansion.com/manufactura/2011/01/10/productos-a-la-medida-de-losmexicanos [Consultado 23 de abril de 2014].

Rojas Colvin, J.; Almagià Flores, A.A.; Ilard, J.S. (2013). Estudio Antropométrico en Párvulos Atendidos por el Sistema Educativo Público Chileno para el Diseño de Mobiliario. Int. J. Morphol., 31, pp. 189-196.

Ruvacalba, A. (2012). Medidas corporales de la población mexicana [Online]. Mexico. [Consultado 2 de mayo de 2014].

Samaniego Riera, D. (2012). Sistema inteligente para reconocimiento de género mediante el sensor Kinect. Universidad Politecnica de Valencia.

Vázquez, L. (2013). Disergonomias por diseño en las escuelas y desarrollo de un proyecto. Cuadernos de la Escuela de Salud Pública, 1, pp. 21-28.

Velardo, C.; Duguelay J.-L. (2011). Real time extraction of body soft biometric from 3d videos. Proceedings of the 19th ACM international conference on Multimedia, 2011. ACM, pp. 781-782.

Vicente Querol M.Á. (2015). Desarrollo de un sistema de captura de siluetas en Android. Proyecto Final de Carrera, Universidad Politécnica de Valencia. Escuela Técnica Superior de Ingenieros de Telecomunicación.

Galindo Sosa, R.V.; Navarrete Modesto, M.; Ocaña Delgado, R.; Gómez Aguirre, M. (2011). Anthropometric Tables Of Industrial Design Students Of UAEM.

Yáñez Mendiola, J. (2009a). Antropometría: mediciones a partir de una cámara fotográfica. Ide@s CONCYTEG, 48.

Revista EIA Rev.EIA.Esc.Ing.Antioq / Universidad EIA 
Yáñez Mendiola, J. (2009b). La antropometría: un primer paso para conocer nuestro entorno. Ide@s CONCYTEG, 48, 2.

Zwane, P.E.; Sithole, M.; Hunter, L. (2010). A preliminary comparative analysis of 3D body scanner, manually taken girth body measurements and size chart measurements. International Journal of Consumer Studies, 34, pp. 265-271.

\section{PARA CITAR ESTE ARTÍCULO / TO REFERENCE THIS ARTICLE / PARA CITAR ESTE ARTIGO /}

Nariño Lescay, R.; Alonso Becerra, A.; Hernández González, A. (2016). Antropometría. Análisis comparativo de las tecnologías para la captación de las dimensiones antropométricas. Revista EIA, 13(26), julio-diciembre, pp. 47-59. [Online]. Disponible en: https://doi.org/10.24050/reia.v13i26.799 